\title{
DETERMINING THE TOP ALL-TIME COLLEGE COACHES THROUGH MARKOV CHAIN-BASED RANK AGGREGATION
}

\author{
MELISSA JAY, VENKATASAI GANESH KARAPAKULA, AND EMMA KRAKOFF
}

\begin{abstract}
We develop a mathematical model that determines the "best all-time college coach(es)" of the past century in a given sport. We propose ranking college coaches through Markov chainbased aggregation of ranked lists using holistic criteria. Our model synthesizes four full or partial ranked lists based on win percentages, victories, career durations, and effort levels to produce the final comprehensive rankings. As a demonstration, we determine that Ron Mason, Augie Garrido, and Gus Donoghue are the top all-time college coaches of the past century in NCAA Division I men's ice hockey, baseball, and men's soccer, respectively. Our general model is applicable not only across all possible sports but also to both male and female coaches. Additionally, it accounts for differences among coaches in their coaching time-periods.
\end{abstract}

\section{CONTEnTs}

1. Introduction $\quad 12$

2. Mathematical Model 13

2.1. Rationale 13

2.2. Methodology 14

2.3. Assumptions 18

3. Ranking Men's Ice Hockey Coaches 19

4. Discussion 22

4.1. Sensitivity Analysis 22

4.2. Strengths 23

4.3. Weaknesses 23

5. Additional Applications 24

5.1. Ranking Baseball Coaches 24

5.2. Ranking Men's Soccer Coaches 24

6. Conclusion 24

Acknowledgments 25

References 25

Appendix 25

\section{INTRODUCTION}

In this paper, we develop a mathematical model that determines, using various criteria, the "best all-time college coach(es)" of the past century in a given sport. Ranking college coaches based on a single factor, such as the win percentage, tends to be biased. For instance, in the 
Men's Ice Hockey Coaching Records of the National Collegiate Athletic Association (NCAA) [4], Alfred Winsor Jr. (1906-1922) is ranked number one on the list of "Winningest Coaches All-Time by Percentage [of Wins]" under "Division I Coaching Records." Although his win percentagethe percentage of wins out of the total games coached - is 0.761 , he has only 86 victories - the number of wins out of the total games coached - compared to Ron Mason (1967-2002), who has 924 victories. Mason is ranked first on the "Winningest Coaches All-Time by Victories" list and third on the "Winningest Coaches All-Time by Percentage [of Wins]" list, whereas Winsor does not even appear on the former list. These rankings neither account for the generational differences between Winsor and Mason's coaching time-periods with respect to the number of total games a college team played nor use holistic criteria. In order to compensate for these deficiencies, we propose ranking college coaches through Markov chain-based rank aggregation. This method allows for various partial ranked lists, which are lists that rank only a select number of coaches, to be aggregated into a single ranked list [1]. To implement this method, we first compile two available partial ranked lists, which are based on win percentages and victories, from the NCAA. Alongside these lists are additional coaching statistics such as years of coaching experience and total number of games coached. Using this data, we generate two new ranked lists - based on years of coaching experience and "effort level" - to expand standards of evaluation. Our model synthesizes these four ranked lists to determine the top five all-time college coaches of the past century in NCAA Division I men's ice hockey, baseball, and men's soccer. Our general model is applicable to all college sports and both genders.

\section{Mathematical Model}

2.1. Rationale. Availability of data is an influential factor when choosing an appropriate model for ranking. It is often difficult and expensive to obtain a complete set of raw data for a full list of college coaches regarding their win percentages, salaries, years of coaching experience across various divisions of a sport, and so on. Although databases of this sort are not usually in the public domain, ranked lists, especially those based on just one criterion, are abundant in this information age. Rank aggregation blends the available partial ranked lists into a more comprehensive ranking. It is an effective technique to create a superior ranking using obtainable data [2].

To aggregate the ranked lists, we use a rating method that involves the "casting of votes" [2] by one individual or item for another. A relatively weak entity in a list casts, according to a criterion, one or more votes for a stronger entity in recognition of the stronger entity's superiority. In this process, every entity obtains a number of votes, attributable to multiple ranked lists. Mathematically manipulating the summation of these votes produces a stochastic matrix, which is also called a transition probability matrix. Graphically, this matrix contains the probabilities of a "random walker" transitioning from every node to every other on a graph that has a node representing every entity. In one's imagination, the proportion of time the random walker spends on each node in the long-run determines the final ranking [2]. The power of this method lies in its pairwise comparison of all entities with one another [1].

The above outlined Markov chain-based method that aggregates both available partial ranked lists and further-generated ranked lists is a particular algorithm motivated by Dwork et al.'s study [1], which compares different methods of rank aggregation. According to the study, rank 
aggregation using Markov processes is effective for comparing partial lists where some entities appear on all the lists and other entities appear only on one or a few lists; that is, this Markov method is effective whenever the partial ranked lists in question are not disjoint. Since we mainly deal with data from multiple lists with some coaches appearing more than once, the Markov method is preferable. Dwork et al. [1] also mention the effectiveness of the Markov method when handling "top- $m$ " lists, which preserve the ranks of only coaches who are ranked within $m$ places in the partial lists and nullify the ranks of the others ranked below $m$. Top- $m$ lists are discussed thoroughly in the next section. The Markov method is both computationally efficient and capable of easily handling partial lists that are widely available on the web [1]. This method is, therefore, practical for ranking the top five all-time college coaches in any sport.

2.2. Methodology. The first step is to rank different coaches under various criteria using available data. Let there be $v$ "data-lists" with different data about $u$ coaches such that $a_{i j}$ is the datum relating to coach $i$ on list $j$. Note that $u, v \in \mathbb{N}$ and $u, v \geq 2$; also, note that the indices $i$ and $j$ belonging to the sets $\mathcal{C}=\{i \in \mathbb{N}: i \leq u\}$ and $\mathcal{L}=\{j \in \mathbb{N}: j \leq v\}$ relate to coaches and data-lists respectively. Then, $v$ ranked lists corresponding to the data-lists are generated as follows: let $r_{i j}$ of the $j$-th ranked list be the ranking of coach $i$ on the data-list $j$; specifically, let $r_{i j}$ be the rank of $a_{i j}$ based on its magnitude relative to the other values in the data-list $j$ such that duplicate values in the data-list $j$ have the same rank. The ranked list $j$ could be in ascending or descending order, but the "best" value(s) in the list $j$ should have a rank of 1.

For example, the first column of Table 1 contains a portion of the list of $u=72$ men's ice hockey coaches in the past century with a minimum of 10 years of coaching experience at the Division I level [4]; the second and the third columns contain $v=2$ data-lists [4] relating to the win percentages and victories respectively, while the fourth and fifth columns contain the $v=2$ ranked lists corresponding to the two data-lists respectively.

\begin{tabular}{|c|c|c|c|c|c|c|}
\hline Coach & Wi. & Vi. & Wi. rank & Vi. rank & T-5 wi. rank & T-5 vi. rank \\
\hline Ron Mason & 0.6961 & 924 & 3 & 1 & 3 & 1 \\
Jerry York & 0.6138 & 913 & 30 & 2 & 0 & 2 \\
Jack Parker & 0.6433 & 873 & 19 & 3 & 0 & 3 \\
$\vdots$ & $\vdots$ & $\vdots$ & $\vdots$ & $\vdots$ & $\vdots$ & $\vdots$ \\
\hline
\end{tabular}

TABLE 1. Win percentage (wi.), victories (vi.) [4], the ranks on the win percentagesbased and victories-based ranked lists - wi. rank and vi. rank respectively - and the corresponding ranks on the top-5 lists - t-5 wi. rank and t-5 vi. rank, respectivelyof each of 72 Division I men's ice hockey coaches. Table 10 in the Appendix is the full version of this table.

Complete sets of desired data concerning all coaches might not always be available. Generally, only data related to a select group of coaches, who are highly ranked in a few of the categories and unranked in the rest, are available. Such "partial lists" of data can skew the final ranking when only the ranked lists are used, as it would ignore the data of the missing coaches. The purpose of the second step is to mitigate this by creating "top- $m$ " lists, which retain the top $m$ ranks but 
render the lower ranks null, as column vectors of a matrix. Let $m \in \mathbb{N}$ be a "cut-off" parameter such that $m \leq u$. Then, let $b_{i j}$ be the entry located on the $i$-th row and the $j$-th column of the $u \times v$ matrix $B$ such that

$$
b_{i j}= \begin{cases}r_{i j} & \text { if } r_{i j} \leq m \\ 0 & \text { if } r_{i j}>m\end{cases}
$$

The sixth and seventh columns of Table 1 , for instance, contain the top- 5 lists, where $m=5$, relating to win percentages and victories of the Division I men's ice hockey coaches respectively.

Depending on the choice of $m$, the matrix $B$, which contains the top- $m$ lists in the form of column vectors, could have one or more rows all filled with 0's, signifying that some of these coaches have ranks below $m$ in all of the categories and thus must not be considered for the final ranking. The purpose of the third step is to eliminate the data of these coaches before proceeding further. Let $u_{0}$ be the number of rows $i$ in $B$ for which $\sum_{j=1}^{v} r_{i j}=0$; that is, let $u_{0}$ be the number of rows $i$ in $B$ having all entries zero. Then, a new matrix $C$ is obtained from $B$ by removing these $u_{0}$ rows as follows. Let $n=u-u_{0}$. Have $c_{s t}$ be the entry located on the $s$-th row and $t$-th column of an $n \times v$ matrix $C$ such that $c_{s t}=b_{i j}$, where $t=j$, for a certain coach $i$, whose ranks in various ranked lists are all on the $s$-th row, given that $\sum_{t=1}^{v} c_{s t} \neq 0$ for every row $s$. In this matrix $C$, each row has at least one nonzero element; that is, each coach represented in this matrix $C$ has a rank within $m$ in at least one of the categories.

The matrix $C$ relating to the top-5 lists for Division I men's ice hockey coaches comprises the second and the third columns of Table 2 .

\begin{tabular}{|c|c|c|}
\hline Coach & T-5 wi. Rank & T-5 vi. Rank \\
\hline Ron Mason & 3 & 1 \\
Jerry York & 0 & 2 \\
Jack Parker & 0 & 3 \\
Rick Comley & 0 & 4 \\
Red Berenson & 0 & 5 \\
Ned Harkness & 2 & 0 \\
Vic Heyliger & 5 & 0 \\
Larry Armstrong & 4 & 0 \\
Alfred Winsor Jr. & 1 & 0 \\
\hline
\end{tabular}

TABLE 2. Win percentages-based and victories-based top-5 lists relating to Division I men's ice hockey coaches.

According to Langville and Meyer [2], "[the] Markov rating method can be summarized with one word: voting." Every column in the matrix $C$ is related to a top- $m$ list, which is further related to a certain ranking under a category. Rankings on every top- $m$ list can be thought of as results of contests between the coaches against one another in a particular category. According to the "results" (or rankings) in that particular category, a higher-ranked coach would receive "vote(s)" on behalf of a lower-ranked coach in acknowledgement of the fact that the higher-ranked coach 
fares better than the lower-ranked coach in that category. The number of votes the higher-ranked coach receives from the lower-ranked coach depends on the number of places the higher-ranked coach is ahead of the lower-ranked one on the corresponding top- $m$ list; this is purely a heuristic. Of course, a lower-ranked coach does not receive any "votes" from a higher-ranked coach, and a coach whose rank is null in that particular category can neither cast "votes" for nor receive them from other coaches. Also, a coach cannot vote for himself. Note that all of these are also heuristics. The fourth step is to record the number of votes the coaches cast for one another in each category. For this purpose, let $d_{t, x y}$ be the entry located on the $x$-th row and the $y$-th column of the $n \times n$ matrix $D_{t}$ (among $v$ such $n \times n$ matrices) such that

$$
d_{t, x y}= \begin{cases}c_{x t}-c_{y t} & \text { if } c_{x t} \geq c_{y t} \geq 1 \\ 0 & \text { otherwise }\end{cases}
$$

in specific, $d_{t, x y}=0$ when $c_{x t}=0$, or when $c_{y t}=0$, or in the case where $1 \leq c_{x t}<c_{y t}$. Thus, $d_{t, x y}$ indicates the theoretical number of votes the $x$-th row's coach casts for the $y$-th column's coach according to the top- $m$ list in the $t$-th column of $C$. In the example of Division I men's ice hockey coaches, the win percentages-based matrix $D_{1}$ and the victories-based matrix $D_{2}$, both of whose rows and columns relate to Ron Mason (RM), Jerry York (JY), Jack Parker (JP), Rick Comley (RC), Red Berenson (RB), Ned Harkness (NH), Vic Heyliger (VH), Larry Armstrong (LA), and Alfred Winsor Jr. (AW) respectively, are as follows:

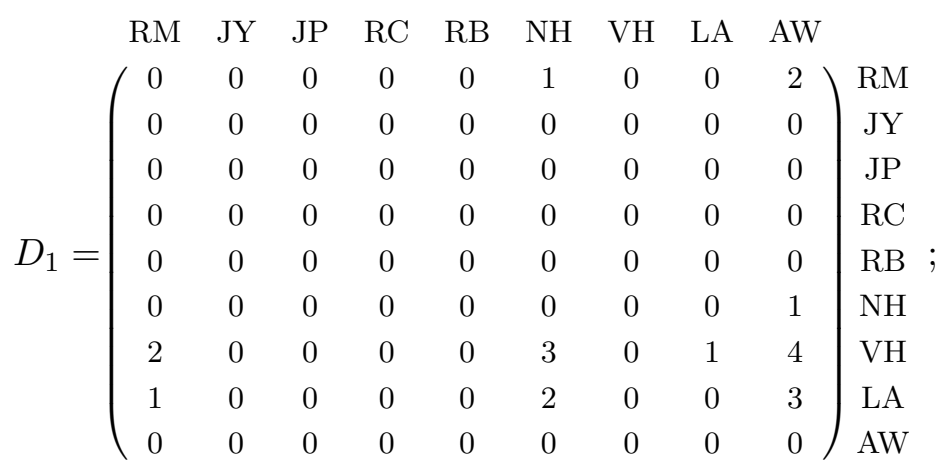

$$
\begin{aligned}
& D_{2}=\left(\begin{array}{ccccccccc|c}
\mathrm{RM} & \mathrm{JY} & \mathrm{JP} & \mathrm{RC} & \mathrm{RB} & \mathrm{NH} & \mathrm{VH} & \mathrm{LA} & \mathrm{AW} \\
1 & 0 & 0 & 0 & 0 & 0 & 0 & 0 & 0 & \mathrm{RM} \\
2 & 0 & 0 & 0 & 0 & 0 & 0 & 0 & 0 & \mathrm{JY} \\
3 & 1 & 0 & 0 & 0 & 0 & 0 & 0 & 0 & \mathrm{JP} \\
4 & 2 & 1 & 0 & 0 & 0 & 0 & 0 & 0 & \mathrm{RC} \\
0 & 0 & 0 & 1 & 0 & 0 & 0 & 0 & 0 & \mathrm{RB} \\
0 & 0 & 0 & 0 & 0 & 0 & 0 & 0 & 0 & \mathrm{VH} \\
0 & 0 & 0 & 0 & 0 & 0 & 0 & 0 & 0 & \mathrm{LA} \\
0 & 0 & 0 & 0 & 0 & 0 & 0 & 0 & 0
\end{array}\right) \text { AW }
\end{aligned}
$$

The fifth step is to aggregate all the votes the coaches cast for one another in all categories. For this, let $Q=\left[q_{g h}\right]_{n \times n}$ with $q_{g h}=\sum_{t=1}^{v} d_{t, g h}$; in other words, $Q=D_{1}+D_{2}+\cdots+D_{v}$. In the previous example, $Q=D_{1}+D_{2}$, and so 


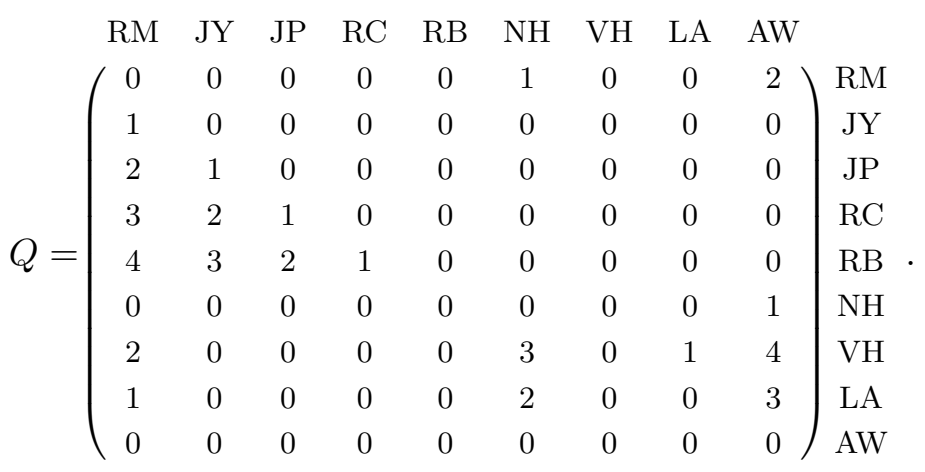

A ranking vector can be teased out from $Q$, the matrix that contains the sum of votes for each candidate under consideration, by first normalizing all the rows of $Q$ to form a stochastic or a substochastic matrix [2]. A sub-stochastic matrix can occur if there is at least one row filled completely with zeros, that is, if at least one coach is unranked in all categories except for one in which he is ranked first. A heuristic can be used to deal with a sub-stochastic matrix: assume that each of the aforementioned coach(es) responsible for the sub-stochastic matrix has a normalized row with equal probabilities under all columns in a right stochastic matrix; this would happen if he were to cast one vote in one category for everyone, including himself. This is obviously an exception to two rules: a coach is not eligible to cast a vote for himself and an unranked coach cannot cast a vote for others. In order to carry out this fifth step of creating the final stochastic matrix, let $\alpha_{g}=\sum_{h=1}^{n} q_{g h}$. Then, let the final left stochastic matrix $S$ be the transpose of $W=\left[w_{e f}\right]_{n \times n}$, an $n \times n$ right stochastic matrix with

$$
w_{e f}= \begin{cases}\frac{q_{e f}}{\alpha_{e}} & \text { if } \alpha_{e} \neq 0 \\ \frac{1}{n} & \text { if } \alpha_{e}=0 .\end{cases}
$$

In the example of Division I men's ice hockey coaches,

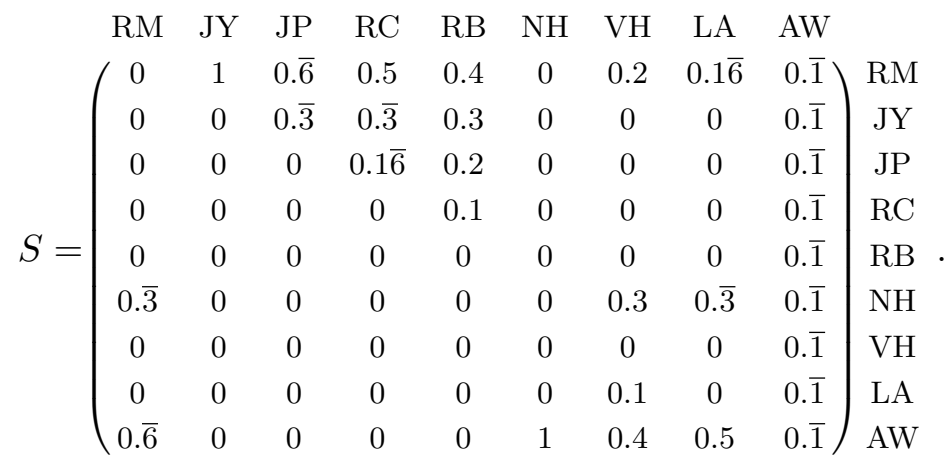

Determining the final rating vector involves solving the eigensystem $S \vec{z}=\vec{z}$ to obtain the stationary probability vector $\vec{z}$, the dominant right eigenvector of $S$ - associated with dominant eigenvalue 1 -whose vector components sum to one. The Perron-Frobenius theorem guarantees the existence and uniqueness of such a stationary probability vector [6]. A variation of a metaphor used by Langville and Meyer [2] explains the intuition behind the usage of stationary probability vector for 
the final ranking of coaches. Suppose a person who is curious about all-time best coaches takes a random walk on a graph constructed using a Markov chain defined by $S$, the left stochastic or transition probability matrix, each element of which represents the probability of transitioning from the node of its column's coach to that of its row's coach. Then, the dominant eigenvector relating to the chain represents the "long-run proportion of the time [the random walker] spends in the states of the chain" [2]. Therefore, the overall rankings are obtained by assigning ranks to the values of $\vec{z}$ in descending order.

For the same example dealing with the two top-5 lists of men's ice hockey coaches, whose Markov graph is shown in Figure 1, Winsor is ranked first, Mason second, Harkness third, York fourth, and Parker fifth, as they tally with the five highest probabilities in the corresponding stationary probability vector

$\vec{z}=\left(\begin{array}{ccccccccl}\text { RM } & \text { JY } & \text { JP } & \text { RC } & \text { RB } & \text { NH } & \text { VH } & \text { LA } & \text { AW } \\ 0.211 & 0.083 & 0.054 & 0.043 & 0.039 & 0.136 & 0.039 & 0.043 & 0.352\end{array}\right)^{T}$.

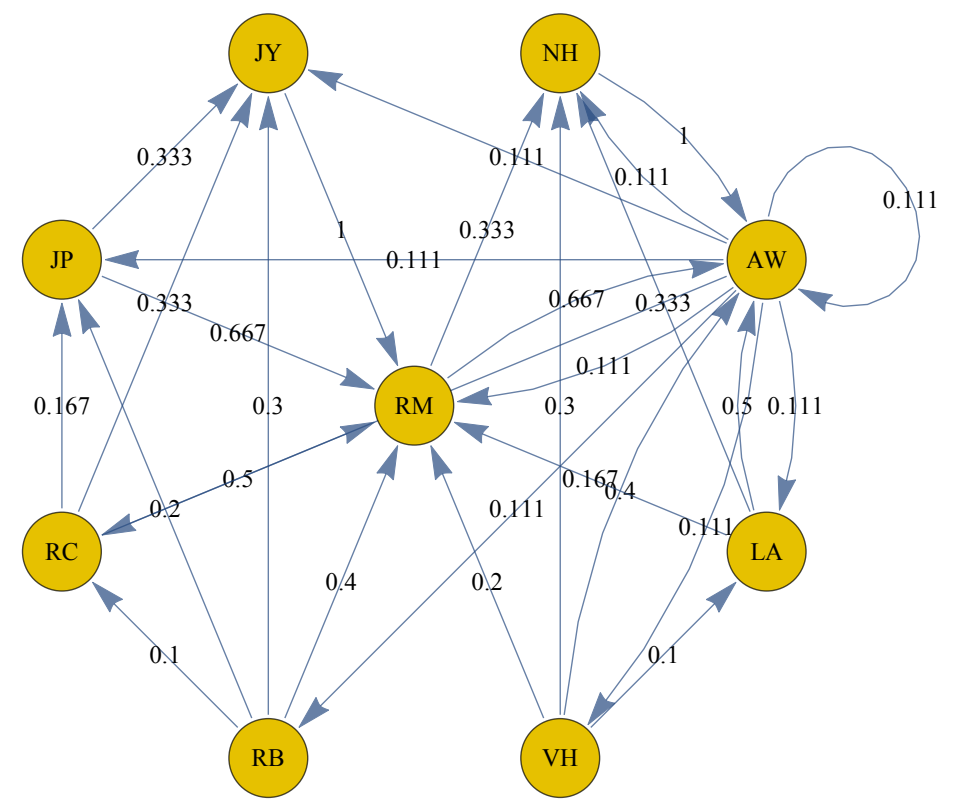

FIgURE 1. Markov graph for the specified example of men's ice hockey coaches.

2.3. Assumptions. The first assumption in the methodology is that the number of votes a higherranked coach receives from a lower-ranked coach is equal to the number of places the higher-ranked coach is ahead of the lower-ranked one in a particular ranking. This assumption is reasonable as it is ideal to place more weight on higher-ranked coaches in the final stochastic matrix. However, this heuristic remains an assumption of the model because each ranking is being thought of as a result of "contests" between the coaches while in reality the coaches in different time periods have not necessarily "competed" against one another. Also, it is possible that the ratings or data (in the data-lists) of some coaches are too close to one another to justify the differences in the rankings. This could occur especially when data such as win percentages are used for ranking. On a related note, we choose to give each ranked list an equal weight. 
The second assumption is that every coach responsible for a sub-stochastic matrix - that is, every coach who is unranked in all the categories except for one category in which he is ranked first-has a "dangling node" [2] that loops back to itself with the same probability that it points toward other nodes. An example of a dangling node is that of Winsor (AW) in Figure 1. Although creation of such node(s) fixes a sub-stochastic matrix, the assumption is that the coach would act as if he cast a vote for everyone, including himself, in one category. This, of course, is a violation of the rules that a coach cannot cast a vote for himself and that an unranked coach cannot cast a vote for others. The assumption, therefore, is that this sort of violation of the rules is acceptable when $Q$ has a row filled with all zeros.

\section{Ranking Men's Ice Hockey Coaches}

To rank the top five all-time coaches in men's ice hockey, we use the college coaching records data published by the NCAA [4]; similar data is available for all college sports. We first select lists that rank past and present coaches who have ten or more years of coaching experience. We choose to consider only the coaches at the Division I level, which is the most competitive level of college sports. The lists rank the coaches by both their victories and win percentages. The data regarding years of coaching experience and total games coached are also available for the coaches on these ranked lists. Since our model strives to show the top five all-time coaches of the past century, we consider only coaches whose careers include at least one year between 1913 and 2013. This procedure renders $u$, the parameter indicating the number of coaches initially considered in the ranking process, a value of 72 .

We first consider a model aggregating two ranked lists, one based on win percentages and another on victories; in this case, $v=2$. The number of victories is the number of wins out of the total games coached. The NCAA [4] defines win percentage of a coach as the division of the sum of victories and half the number of ties by the total number of games coached.

Ranking using win percentages is a valuable way to compare coaches who have coached a similar number of games. However, Alfred Winsor Jr. ranks number one on the win percentages-based ranked list despite having coached far fewer games than the other coaches on the same list. Figure 2 is a graph of the total number of games $(T)$ coached by every listed inactive coach plotted against the last year $(L)$ of his career. The exponentially fitted curve for this data is $T=10^{-17} e^{0.0229 L}$ with an $R^{2}$ of 0.7067 . This moderately strong coefficient of determination implies that the number of games coached in one's career has increased almost exponentially over time.

To balance this bias, we aggregate the win percentages-based ranked list with the victoriesbased ranked list. We do this by first creating two top-50 lists, which have $m=50$, using the aforementioned ranked lists. Since Winsor won only a few games during his career (1906-1922) in comparison with the other coaches, his rank is nullified - that is, his rank is assigned a value of zero - on the victories-based top-50 list. Then, using the Markov process to combine the two top-50 lists eliminates much of the bias associated with each ranked list published by the NCAA. As a consequence, Winsor, Harkness, Mason, Berenson, and York emerge as the top five coaches in the final ranking. Table 3 shows the ranks on win percentages-based and victories-based top-50 lists, represented by t-50 wi. rank and t-50 vi. rank respectively, along with the overall rank of each of these top five coaches. 


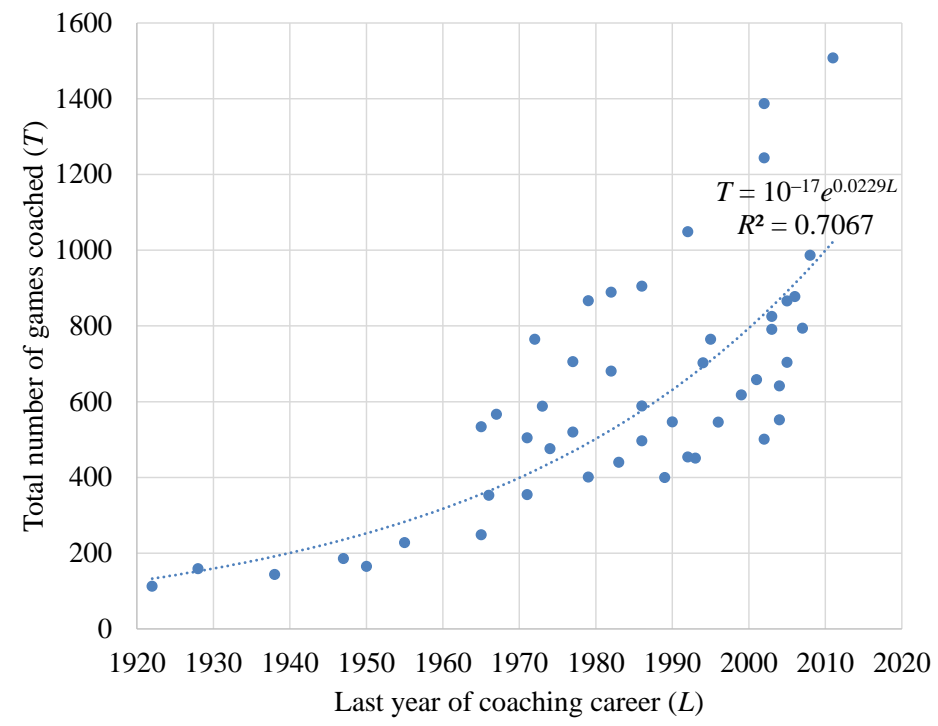

Figure 2. Total games of inactive coaches versus their retirement timings [4].

\begin{tabular}{|c|c|c|c|}
\hline Coach & T-50 wi. rank & T-50 vi. rank & Overall rank \\
\hline AW & 1 & 0 & 1 \\
NH & 2 & 27 & 2 \\
RM & 3 & 1 & 3 \\
RB & 10 & 5 & 4 \\
JY & 30 & 2 & 5 \\
\hline
\end{tabular}

TABle 3. Top five all-time Division I men's ice hockey coaches determined using win percentages-based and victories-based top-50 lists.

This aggregated ranking gives Winsor preference because he is the sole coach to appear on only one of the two lists. Graphically, this result is intuitive. Winsor casts no votes for the others, and Mason (ranked first in victories) ranks two places below Winsor in win percentage. As a result, Mason casts two votes for Winsor. Since many votes are cast for Mason, whenever Mason's node is reached on the Markov chain, there is a high probability that Winsor's node is the next destination. Table 3 also illustrates the inherent power of the Markov method in comparing all coaches pairwise with one another: although Harkness is ranked twenty seventh on the victories-based ranked list, he fares better than Mason in the win percentages-based "contest;" this consequently gives Harkness an edge in the overall rankings.

To diversify preferences in rank aggregation and explore more complex models, we increase $v$; this is achieved by manipulating available data and generating more data-lists as a result. We first increase $v$ to 3 by including a ranked list based on years of coaching experience, presuming that in general coaches with longer careers are more "experienced" and better than coaches with shorter terms in Division I. This is a generated list; it is derived from the NCAA-published data [4] on coaching experience pertaining to the coaches on the two available win percentages-based and victories-based ranked lists, because the NCAA does not separately publish a complete set of data 
regarding career durations. Furthermore, we observe no significant correlation between $L$ and years of coaching experience, so incorporating this ranked list into the aggregated model does not induce any obvious partiality. When career durations are also considered, making $v=3$, while $m$ is still 50, Mason, Winsor, York, Harkness, and Parker are ranked first through fifth overall, respectively. Table 4 displays the t-50 wi. rank, the t-50 vi. rank, and the t-50 ex. rank - the rank on the coaching experience-based top-50 list - along with the overall rank of each of these top five coaches.

\begin{tabular}{|c|c|c|c|c|}
\hline Coach & T-50 wi. rank & T-50 vi. rank & T-50 ex. rank & Overall rank \\
\hline RM & 3 & 1 & 4 & 1 \\
AW & 1 & 0 & 0 & 2 \\
JY & 30 & 2 & 1 & 3 \\
NH & 2 & 27 & 28 & 4 \\
JP & 19 & 3 & 2 & 5 \\
\hline
\end{tabular}

TABle 4. Top five all-time Division I men's ice hockey coaches determined using win percentages-based, victories-based, and experience-based top-50 lists.

While coaching experience is a good criterion for ranking, there may be huge differences in the total games coached among coaches with a similar number of years coached. This is especially true in the case of men's ice hockey coaches, as can be seen in Figure 2, which shows that there has been a tremendous increase in the workload and responsibility for coaches over time. To address this concern, we increase $v$ to 4 by creating a fourth data-list based on a new measure called "effort level." We define effort level as the division of the total number of games coached by the number of years of coaching experience. This data-list approximates the average number of games coached per year for each coach. When the data-list containing effort levels is also used in the final ranking process, making $v=4$, while $m$ equals 50, Mason, Berenson, York, Winsor, and Parker are ranked first through fifth overall, respectively. Figure 3 shows the Markov graph for this process, which synthesizes win percentages-based, victories-based, coaching experience-based, and effort levelsbased top-50 lists. Table 5 displays the t-50 wi. rank, the t-50 vi. rank, the t-50 ex. rank, and the t-50 ef. rank - the rank on the effort level-based top-50 list - along with the overall ranking of each of the top five coaches. Table 6 presents the win percentage (wi.), victories (vi.), years of coaching experience (ex.), and effort level (ef.) of each of the top five coaches.

\begin{tabular}{|c|c|c|c|c|c|}
\hline Coach & T-50 wi. rank & T-50 vi. rank & T-50 ex. rank & T-50 ef. rank & Overall rank \\
\hline RM & 3 & 1 & 4 & 20 & 1 \\
RB & 10 & 5 & 12 & 2 & 2 \\
JY & 30 & 2 & 1 & 15 & 3 \\
AW & 1 & 0 & 0 & 0 & 4 \\
JP & 19 & 3 & 2 & 24 & 5 \\
\hline
\end{tabular}

TABLE 5. Top five all-time Division I men's ice hockey coaches determined using win percentages-, victories-, coaching experience-, and effort levels-based top-50 lists. 


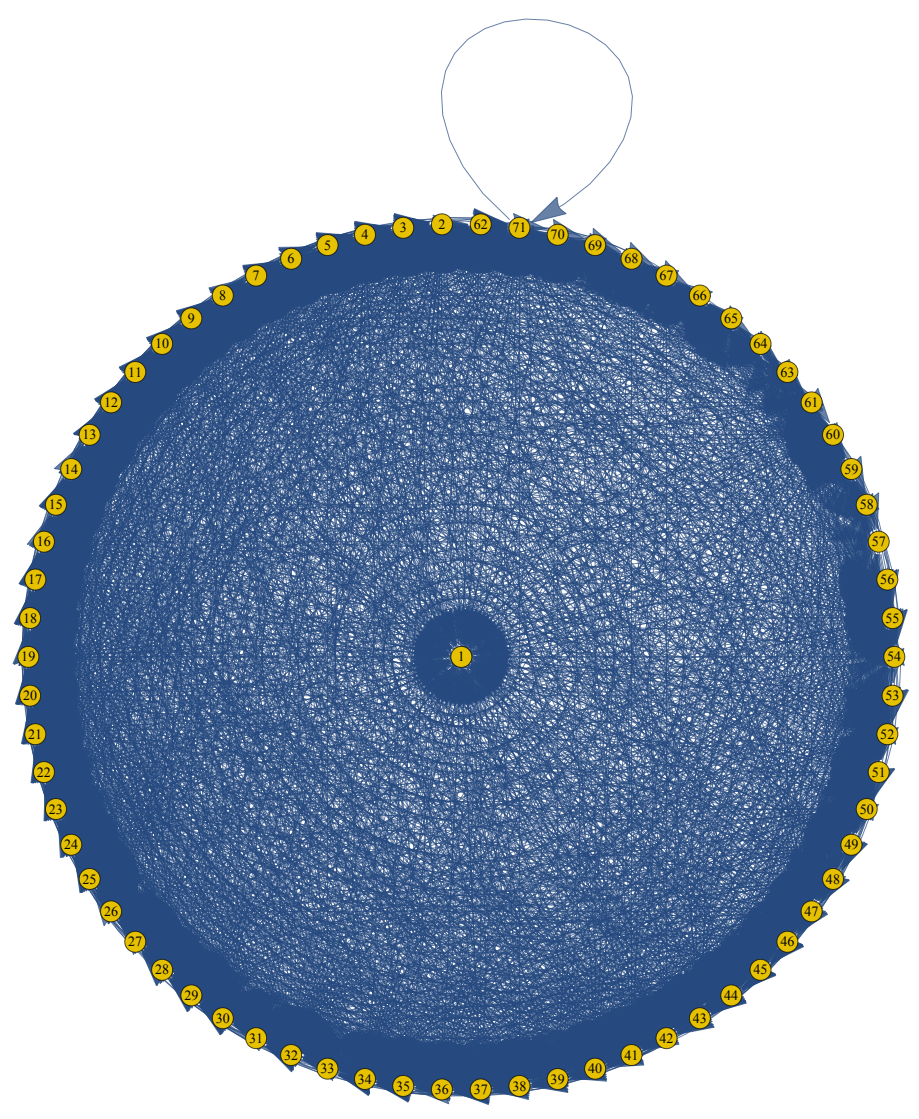

FiguRE 3. Markov graph related to synthesis of the four top-50 lists based on holistic criteria for Division I men's ice hockey coaches.

\begin{tabular}{|c|c|c|c|c|c|}
\hline Overall rank & Coach & Wi. & Vi. & Ex. & Ef. \\
\hline 1 & Ron Mason (1967-2002) & 0.6961 & 924 & 36 & 38.53 \\
2 & Red Berenson (1985-2012) & 0.6693 & 752 & 28 & 42.18 \\
3 & Jerry York (1973-2012) & 0.6138 & 913 & 40 & 39.10 \\
4 & Alfred Winsor Jr. (1906-1922) & 0.7611 & 86 & 13 & 8.69 \\
5 & Jack Parker (1974-2012) & 0.6433 & 873 & 39 & 37.06 \\
\hline
\end{tabular}

TABle 6. Statistics [4] of the top five all-time Division I men's ice hockey coaches determined using win percentages-, victories-, coaching experience-, and effort levelsbased top-50 lists.

\section{Discussion}

4.1. Sensitivity Analysis. We test the robustness of our model by varying the parameter $m$. Table 7 shows the top five coaches resulting from Markov chain-based aggregation of win percentagesbased, victories-based, coaching experience-based, and effort levels-based ranked lists relating to Division I men's ice hockey coaches for different values of $m$. 
JAY, KARAPAKULA, AND KRAKOFF

\begin{tabular}{|c|c|c|c|c|c|c|c|}
\hline Rank & $m=10$ & $m=20$ & $m=30$ & $m=40$ & $m=50$ & $m=60$ & $m=70$ \\
\hline 1 & $\mathrm{RM}$ & $\mathrm{AW}$ & $\mathrm{RM}$ & $\mathrm{RM}$ & $\mathrm{RM}$ & $\mathrm{RM}$ & $\mathrm{RM}$ \\
2 & $\mathrm{JY}$ & $\mathrm{RM}$ & $\mathrm{AW}$ & $\mathrm{RB}$ & $\mathrm{RB}$ & $\mathrm{RB}$ & $\mathrm{RB}$ \\
3 & $\mathrm{AW}$ & $\mathrm{RB}$ & $\mathrm{RB}$ & $\mathrm{AW}$ & $\mathrm{JY}$ & $\mathrm{JY}$ & $\mathrm{JY}$ \\
4 & $\mathrm{JP}$ & $\mathrm{DW}$ & $\mathrm{NH}$ & $\mathrm{JY}$ & $\mathrm{AW}$ & $\mathrm{AW}$ & $\mathrm{JP}$ \\
5 & $\mathrm{NH}$ & $\mathrm{NH}$ & $\mathrm{DW}$ & $\mathrm{DW}$ & $\mathrm{JP}$ & $\mathrm{JP}$ & $\mathrm{RC}$ \\
\hline
\end{tabular}

TABLE 7. Top five all-time Division I men's ice hockey coaches determined using win percentages-based, victories-based, experience-based, and effort levels-based top- $m$ lists for different values of $m$. Note that DW refers to Doug Woog.

In Table 7, Mason (RM) is almost always ranked first, and Berenson (RB) is consistently ranked second for $m=40,50,60,70$. York (JY) is also steadily ranked third when top-50, top-60, and top-70 lists are used. This pattern indicates that the rankings of the top coaches become consistent as $m$ gets closer to its largest possible value, although there are subtle positional changes elsewhere. Moreover, even for seven different values of $m$, the top five positions are always filled by five of the eight names, namely, AW, DW, JP, JY, RB, RC, RM, and NH. This suggests that there are only minor changes in the top collection of coaches for different values of $m$. Therefore, our model is fairly robust to changes in $m$.

4.2. Strengths. As evinced in the sensitivity analysis, our model is fairly robust. This model can easily handle ranked lists of unequal lengths, providing a way to combine partial ranked lists with computational efficiency [1]. Moreover, the mathematical model is applicable even when a complete set of raw data is available. Since the NCAA provides limited data regarding coaches in all college sports, this model has a fair degree of generality. Furthermore, the methodology does not suggest any gender-specific measures, so this model is applicable in ranking all coaches, regardless of their genders, of a chosen sport. The model accounts for differences among coaches in their coaching time-periods by using win percentages along with victories as criteria in rank aggregation. In addition, incorporating career durations-based and effort levels-based ranked lists into the final ranking allows for the appreciation of coaches with relatively more coaching experience and those who coached relatively more games per year, respectively. Thus, our model uses holistic criteria for ranking.

4.3. Weaknesses. Our model does not take into account the top coaches based purely on years of experience and on effort levels, because the corresponding lists are generated using the other ranked lists. As a consequence, we might be excluding some top coaches in the categories of coaching experience and effort level. In addition, the model disadvantages active coaches, whose careers are not yet over, as their data does not reflect their future potential. However, this is acceptable because the aim of the model is to rank the top "all-time" coaches. Consideration of active coaches in the model could be seen as accounting for the possibility that some of them could have already outpaced the inactive ones in some categories. 


\section{Additional Applications}

5.1. Ranking Baseball Coaches. Table 8 presents the the win percentage (wi.), victories (vi.), years of coaching experience (ex.), and effort level (ef.) of each of the top five all-time Division I baseball coaches. This list is determined using win percentages-based, victories-based, coaching experience-based, and effort levels-based top-50 lists obtained from the baseball coaching records published by the NCAA [3]. Augie Garrido emerges as the best coach.

\begin{tabular}{|c|c|c|c|c|c|}
\hline Overall rank & Coach & Wi. & Vi. & Ex. & Ef. \\
\hline 1 & Augie Garrido (1969-2012) & 0.6849 & 1847 & 44 & 61.43 \\
2 & John Barry (1921-1960) & 0.8071 & 619 & 40 & 19.25 \\
3 & Gene Stephenson (1978-2012) & 0.7351 & 1798 & 35 & 69.94 \\
4 & Mike Martin (1980-2012) & 0.7432 & 1723 & 33 & 70.33 \\
5 & Cliff Gustafson (1968-1996) & 0.7925 & 1427 & 29 & 62.14 \\
\hline
\end{tabular}

TABLE 8. Statistics [3] of the top five all-time Division I baseball coaches determined using win percentages-based, victories-based, coaching experience-based, and effort levels-based top-50 lists.

5.2. Ranking Men's Soccer Coaches. Table 9 presents the the win percentage (wi.), victories (vi.), years of coaching experience (ex.), and effort level (ef.) of each of the top five all-time Division I men's soccer coaches. This list is determined using win percentages-based, victoriesbased, coaching experience-based, and effort levels-based top-50 lists obtained from the men's soccer coaching records published by the NCAA [5]. Gus Donoghue comes forth as the top "alltime" men's soccer coach.

\begin{tabular}{|c|c|c|c|c|c|}
\hline Overall rank & Coach & Wi. & Vi. & Ex. & Ef. \\
\hline 1 & Gus Donoghue (1946-1960) & 0.8707 & 121 & 15 & 9.8 \\
2 & Jerry Yeagley (1973-2003) & 0.8210 & 544 & 31 & 22.26 \\
3 & Stephen Negoesco (1962-2000) & 0.7365 & 540 & 39 & 19.95 \\
4 & Jack Mackenzie (1969-2011) & 0.6518 & 516 & 43 & 19.77 \\
5 & Sigi Schmid (1980-1998) & 0.8098 & 322 & 19 & 22 \\
\hline
\end{tabular}

TABLE 9. Statistics [5] of the top five all-time Division I men's soccer coaches determined using win percentages-based, victories-based, coaching experience-based, and effort levels-based top-50 lists.

\section{Conclusion}

The final results of the model that uses top-50 lists are as follows. The top five NCAA Division I men's ice hockey coaches are Ron Mason, Red Berenson, Jerry York, Alfred Winsor Jr., and Jack Parker. The top five NCAA Division I baseball coaches are Augie Garrido, John Barry, Gene Stephenson, Mike Martin, and Cliff Gustafson. The top five NCAA Division I men's soccer coaches are Gus Donoghue, Jerry Yeagley, Stephen Negoesco, Jack Mackenzie and Sigi Schmid. This model 
is applicable to coaches of all college sports and both genders. It also accounts for differences among coaches in their coaching time-periods. Sensitivity analysis reveals that our model, whose key feature is pairwise comparison and rank aggregation using Markov processes, is fairly robust.

Our model also theoretically extends to account for the possibility that all the ranking criteria may not be equally important. In the algorithm we suggest, the matrix of aggregated votes, $Q$, is simply $\sum_{i=1}^{v} D_{i}$, where $D_{i}$ is the matrix of votes based on the top- $m$ list of ranked list $i$. This assumes that all the ranking criteria have equal importance. This restriction is relaxed by letting $Q=\sum_{i=1}^{v} \gamma_{i} D_{i}$, where $\gamma_{1}, \gamma_{2}, \ldots, \gamma_{v} \in \mathbb{R}^{+}$, as an alternative, thus enabling a flexible weighting of the ranked lists. Further work could explore more generalizations of our model.

\section{ACKNOWLEDGMENTS}

This paper earned our team the designation of Finalist in the Mathematical Contest in Modeling held in 2014 by the Consortium for Mathematics and Its Applications. We are grateful to Professor Amelia Taylor for coaching and supporting us. We are also thankful to the Colorado College Department of Mathematics and Computer Science for funding us to participate in the contest.

\section{REFERENCES}

1. Dwork, Cynthia, Ravi Kumar, Moni Naor, and D. Sivakumar. "Rank Aggregation Methods for the Web." In Proceedings of the 10th International Conference on World Wide Web, 613-622. New York: Association for Computing Machinery, 2001. http://dx.doi.org/10.1145/371920.372165.

2. Langville, Amy N., and Carl D. Meyer. Who's \#1?: The Science of Rating and Ranking. Princeton, New Jersey: Princeton University Press, 2012.

3. National Collegiate Athletic Association. 2013. "Baseball Coaching Records."

http://fs.ncaa.org/Docs/stats/baseball_RB/2013/Coaching.pdf.

4. National Collegiate Athletic Association. 2013. "Men's Ice Hockey Coaching Records."

http://fs.ncaa.org/Docs/stats/m_icehockey_rb/2013/Coaches.pdf.

5. National Collegiate Athletic Association. 2013. "Men's Soccer Coaching Records."

http://fs.ncaa.org/Docs/stats/m_soccer_RB/2013/coaches.pdf.

6. Sternberg, Shlomo. Dynamical Systems. Mineola, New York: Dover Publications, 2010.

\section{APPENDIX}

\begin{tabular}{|c|c|c|c|c|c|c|}
\hline Coach & Wi. & Vi. & Wi. rank & Vi. rank & T-5 wi. rank & T-5 vi. rank \\
\hline Ron Mason & 0.6961 & 924 & 3 & 1 & 3 & 1 \\
Jerry York & 0.6138 & 913 & 30 & 2 & 0 & 2 \\
Jack Parker & 0.6433 & 873 & 19 & 3 & 0 & 3 \\
Rick Comley & 0.5557 & 783 & 46 & 4 & 0 & 4 \\
Red Berenson & 0.6693 & 752 & 10 & 5 & 0 & 5 \\
Len Ceglarski & 0.6578 & 672 & 14 & 6 & 0 & 0 \\
Jeff Sauer & 0.5494 & 655 & 51 & 7 & 0 & 0 \\
Mike McShane & 0.6489 & 610 & 16 & 8 & 0 & 0 \\
Don Lucia & 0.6331 & 596 & 22 & 9 & 0 & 0 \\
George Gwozdecky & 0.5951 & 573 & 38 & 10 & 0 & 0 \\
John Maclnnes & 0.6462 & 555 & 17 & 11 & 0 & 0 \\
Jack Riley & 0.6099 & 542 & 31 & 12 & 0 & 0 \\
\hline
\end{tabular}




\begin{tabular}{|c|c|c|c|c|c|c|}
\hline Coach & Wi. & Vi. & Wi. rank & Vi. rank & T-5 wi. rank & T-5 vi. rank \\
\hline John Kelley & 0.6647 & 501 & 12 & 13 & 0 & 0 \\
\hline Richard Umile & 0.6359 & 497 & 21 & 14 & 0 & 0 \\
\hline Joe Marsh & 0.5333 & 482 & 57 & 15 & 0 & 0 \\
\hline Murray Armstrong & 0.6735 & 460 & 9 & 16 & 0 & 0 \\
\hline Doug Ross & 0.6026 & 457 & 34 & 17 & 0 & 0 \\
\hline Bill Wilkinson & 0.4838 & 437 & 64 & 18 & 0 & 0 \\
\hline Rick Gotkin & 0.5800 & 434 & 43 & 19 & 0 & 0 \\
\hline Shawn Walsh & 0.6786 & 423 & 7 & 20 & 0 & 0 \\
\hline John Gasparini & 0.5974 & 407 & 37 & 21 & 0 & 0 \\
\hline Mike Gilligan & 0.5442 & 407 & 53 & 21 & 0 & 0 \\
\hline Craig Dahl & 0.5029 & 407 & 60 & 21 & 0 & 0 \\
\hline Bob Johnson & 0.6160 & 406 & 28 & 24 & 0 & 0 \\
\hline Doug Woog & 0.6634 & 390 & 13 & 25 & 0 & 0 \\
\hline Amo Bessone & 0.4591 & 387 & 71 & 26 & 0 & 0 \\
\hline Ned Harkness & 0.7404 & 380 & 2 & 27 & 2 & 0 \\
\hline Mike Sertich & 0.4867 & 375 & 63 & 28 & 0 & 0 \\
\hline Charlie Holt & 0.6307 & 364 & 23 & 29 & 0 & 0 \\
\hline Tim Taylor & 0.4356 & 354 & 72 & 30 & 0 & 0 \\
\hline Don Cahoon & 0.4684 & 346 & 69 & 31 & 0 & 0 \\
\hline Jeff Jackson & 0.6767 & 342 & 8 & 32 & 0 & 0 \\
\hline Brian Cavanaugh & 0.5249 & 341 & 58 & 33 & 0 & 0 \\
\hline Rand Pecknold & 0.5853 & 337 & 41 & 34 & 0 & 0 \\
\hline Bob Daniels & 0.4819 & 334 & 65 & 35 & 0 & 0 \\
\hline Mike Schafer & 0.6300 & 332 & 24 & 36 & 0 & 0 \\
\hline Bruce Marshall & 0.4741 & 332 & 66 & 36 & 0 & 0 \\
\hline Jerry Welsh & 0.4654 & 328 & 70 & 38 & 0 & 0 \\
\hline William J. Cleary Jr. & 0.6143 & 325 & 29 & 39 & 0 & 0 \\
\hline Vic Heyliger & 0.6859 & 318 & 5 & 40 & 5 & 0 \\
\hline Dean Blais & 0.6437 & 317 & 18 & 41 & 0 & 0 \\
\hline Ralph Weiland & 0.6426 & 316 & 20 & 42 & 0 & 0 \\
\hline Tim Whitehead & 0.5549 & 315 & 47 & 43 & 0 & 0 \\
\hline Don Vaughan & 0.5007 & 314 & 62 & 44 & 0 & 0 \\
\hline Bob Gaudet & 0.4720 & 312 & 68 & 45 & 0 & 0 \\
\hline Edward J. Jeremiah & 0.5538 & 308 & 48 & 46 & 0 & 0 \\
\hline Mark Morris & 0.6527 & 306 & 15 & 47 & 0 & 0 \\
\hline Rob Riley & 0.5031 & 306 & 59 & 47 & 0 & 0 \\
\hline Frank Serratore & 0.4730 & 304 & 67 & 49 & 0 & 0 \\
\hline Mark Mazzoleni & 0.5861 & 302 & 40 & 50 & 0 & 0 \\
\hline Scott Owens & 0.6082 & 299 & 32 & 51 & 0 & 0 \\
\hline Dick Bertrand & 0.6066 & 292 & 33 & 52 & 0 & 0 \\
\hline Al Renfrew & 0.5017 & 288 & 61 & 53 & 0 & 0 \\
\hline Brush Christiansen & 0.5531 & 287 & 49 & 54 & 0 & 0 \\
\hline Enrico Blasi & 0.5985 & 286 & 36 & 55 & 0 & 0 \\
\hline
\end{tabular}




\begin{tabular}{|c|c|c|c|c|c|c|}
\hline Coach & Wi. & Vi. & Wi. rank & Vi. rank & T-5 wi. rank & T-5 vi. rank \\
\hline Murray Murdoch & 0.5393 & 278 & 55 & 56 & 0 & 0 \\
Terry Slater & 0.5782 & 251 & 44 & 57 & 0 & 0 \\
Lou Lamoriello & 0.5716 & 244 & 45 & 58 & 0 & 0 \\
Thom Lawler & 0.6172 & 242 & 27 & 59 & 0 & 0 \\
Brad Buetow & 0.5510 & 239 & 50 & 60 & 0 & 0 \\
Mike Addesa & 0.6013 & 236 & 35 & 61 & 0 & 0 \\
Mike Eaves & 0.5492 & 218 & 52 & 62 & 0 & 0 \\
George Menard & 0.5944 & 204 & 39 & 63 & 0 & 0 \\
John Mariucci & 0.5836 & 197 & 42 & 64 & 0 & 0 \\
Tom Serratore & 0.5388 & 195 & 56 & 65 & 0 & 0 \\
Olav Kollevoll & 0.6225 & 153 & 26 & 66 & 0 & 0 \\
Cheddy Thompson & 0.6689 & 150 & 11 & 67 & 0 & 0 \\
Larry Armstrong & 0.6882 & 123 & 4 & 68 & 4 & 0 \\
Clarence Wanamaker & 0.6258 & 97 & 25 & 69 & 0 & 0 \\
Joseph Stubbs & 0.6806 & 95 & 6 & 70 & 0 & 0 \\
J. Howard Starr & 0.5394 & 87 & 54 & 71 & 0 & 0 \\
Alfred Winsor Jr. & 0.7611 & 86 & 1 & 72 & 1 & 0 \\
\hline 1O. Win perce
\end{tabular}

TABle 10. Win percentage (wi.), victories (vi.) [4], the ranks on the win percentages-based and victories-based ranked lists-wi. rank and vi. rank respectively - and the corresponding ranks on the top-5 lists - t-5 wi. rank and t-5 vi. rank, respectively - of each of 72 Division I men's ice hockey coaches.

(Jay) Colorado College, 902 N. Cascade Ave., Colorado Springs, CO 80946, USA

E-mail address: melissa.jay@coloradocollege.edu

(Karapakula) Colorado College, 902 N. Cascade Ave., Colorado Springs, CO 80946, USA

E-mail address: vgkarapakula@coloradocollege.edu

(Krakoff) Colorado College, 902 N. Cascade Ave., Colorado Springs, CO 80946, USA

E-mail address: emma.krakoff@coloradocollege.edu 